\title{
Time-frequency equivalence in removing sinusoidal interference from electrocardiograms
}

\author{
Brandon S. Coventry ${ }^{1}$, Cecil W. Thomas ${ }^{2}$ \\ ${ }^{1}$ Department of Electrical \& Computer Engineering, Saint Louis University, 3450 Lindell Blvd., St. Louis, MO, 63103, USA \\ ${ }^{2}$ Department of Biomedical Engineering, Saint Louis University, 3507 Lindell Blvd., St. Louis, MO 63103, USA
}

Email address:

cecil2@icloud.com (C. W. Thomas)

\section{To cite this article:}

Brandon S. Coventry, Cecil W. Thomas. Time-Frequency Equivalence in Removing Sinusoidal Interference from Electrocardiograms. International Journal of Biomedical Science and Engineering. Vol. 2, No. 4, 2014, pp. 27-32. doi: 10.11648/j.ijbse.20140204.11

\begin{abstract}
In recorded bioelectric signals, such as the electrocardiogram, sinusoidal interference from power lines or other sources causes distortion in the signal and may lead to misdiagnosis. For long or continuous recordings, adaptive filtering can be effective in minimizing the interference. For short recording, the options are limited. Subtractive methods have been used, but they do not distinguish between the interference and signal components with similar frequency. A new method can distinguish between signal and interference, so that the interference can be removed with very small residual error. In clinical recordings, the frequency of powerline interference is known, but the adaptive nature of the algorithm allows extension to cases when the frequency of interference is not known exactly.
\end{abstract}

Keywords: Interference Removal, Electrocardiogram, Time-Frequency, Filtering

\section{Introduction}

In many biopotential measurement applications, a recorded signal will contain interference or noise components that degrade and otherwise alter the signal in an unacceptable way. This noise is often due to power line interference (PLI), which is typically a noise of known frequency but unknown amplitude and phase. The interference may also consist of multiple sinusoids that may be harmonically related, as in the case of PLI, or non-harmonically related. In [1], a thorough model characterizes the sources of noise in recorded signals including power line interference and electromagnetic interference inherent in electrodes. Canceling this noise is of interest for a wide range of biosignal applications [2, 3], but this discussion will focus on the ECG.

The electrocardiogram (ECG) is a diagnostic tool that can be valuable in the detection and analysis of arrhythmias, structural abnormalities, and metabolic deficits such as ischemia [4,5]. The normal ECG also has an easily recognizable shape that is ideal for the evaluation of noise removal algorithms. Some typical ECG signals are available in an MIT-BIH physiological signals database [6].

Notch or lowpass filters can be utilized when the frequency of interference and its harmonics are at higher frequencies than that of the signal. Chebyshev type II and Nyquist filters have both a low pass characteristic for conditioning low frequency signals and a notch at the frequency of interference $[7,8]$. However, when the interference frequencies occupy the same bandwidth as the frequency components of the signal of interest, low pass and notch filtering do not produce satisfactory results. These filters will distort the amplitude and phase of the desired signal components, resulting in detrimental effects on the waveform. They will also become ineffective if the interference frequency varies even slightly. More importantly, the filters have no mechanism for distinguishing signal from interference.

Signal averaging, normally applied to signals corrupted by random noise [9], may attenuate a coherent interference, but fails to provide satisfactory noise cancelation [10,11]. Combining linear filtering with signal averaging noise reduction techniques forms a filter residue method [12], which defines a residue as the difference between the input signal and the corresponding signal average at every point. The residue is passed through a low pass filter and the result is added back into the signal average. The method appears to work better than general low pass filtering or median filtering methods; however it requires a relatively large data segment to obtain an accurate signal average and timing characteristics of the $\mathrm{R}$ wave are necessary, making this method less effective for short time segments.

When the frequency of interference is stationary, sinusoidal, and has a relatively large amount of energy, spectral 
interpolation can be used. First, the spike in the frequency spectrum corresponding to the noise component is removed, and then the missing value is replaced using interpolation. While the results can be partially successful, the results are often not acceptable in the discrete case due to spectral leakage. Alternatively, interference can be estimated in time or frequency using interpolation, and then subtracting the estimate from the original signal. This method only works when discontinuities occur at predictable locations in the non-windowed spectrum, and the frequencies of interference are significantly far from the frequencies of the signal [13].

Many adaptive methods have also been used to remove noise from ECG signals. Adaptive notch filtering, whose parameters vary based on previous filter outputs, have been developed by several groups for tracking, estimating, and rejecting noise in a signal. A Kalman filter for ECG signal enhancement [14], assumes that the ECG is normally distributed, and uses the time evolution of the ECG signal to adapt filter parameters to correct the frequency of interference. An $\mathrm{H} \infty$ filter to denoise an ECG signal [15] has two advantages over the Kalman filters: there is no need to make assumptions about the statistical properties of the input signal and it is more robust.

An adaptive notch filtering in a dynamic system model [16] can determine the amplitude, initial, and total phases of the sinusoidal interference. A nonlinear adaptive notch filtering scheme [17] extracts the interference without the need for a power reference signal. A phase locked loop can also be used in power line interference cancelation [18]. While adaptive systems are very robust and accurate, a certain settling time is required for the filter to stabilize and accurately remove the interference. An adaptive sinusoid interference cancelation system seeks to minimize settling time [19]. While mid to long length data segments may have enough time for a system to stabilize, short time segments often do not, making the adaptive algorithm methods ineffective.

Time-frequency analysis, especially using wavelets, has also been of interest in ECG noise elimination. Wavelet decomposition, which creates sparse representations for piecewise regular signals including their transients and singularities, has been used in applications such as signal acquisition, compression, and denoising applications [20-22]. Wavelet techniques include binary wavelet denoising [23, a dynamic denoising [24], and nonlinear thresholding [25] to denoise the ECG. In general, wavelets provide an excellent means of denoising the ECG, but suffer in terms of computational complexity.

Subtraction methods simply determine sinusoidal parameters, such as amplitude, frequency, and phase, and then subtract the sinusoidal components from the original signal. Finding these sinusoidal components, however, is not trivial, especially when the source of the noise has unknown amplitude and phase, which is usually the case. One method of determining sinusoidal parameters in noise components uses a weighted finite Fourier integral to determine frequency [26]. Once frequency has been determined, the method uses a least squares approximation with the derived Fourier coefficients to determine amplitude and phase. The subtraction procedure can be generalized to remove exponential (including sinusoidal) interference [27]. For this method, the interference must obey certain orthogonality conditions, and good performance requires that the interference contain an integer number of cycles. In the worst case, the method fails to denoise the signal. In [28], a novel method uses Legendre moments to remove noise from a signal. The method can be effective when the low and high frequencies are uncorrelated.

In biomedical applications, bioelectric sensing devices often send their acquired signals through high gain amplifiers with very high common mode rejection ratios (CMMR). Electrode impedances, cable mismatch, and stray power line coupling introduce common mode voltage that cannot be suppressed with high CMRR [1,29]. Several subtraction methods have been developed to handle this interference situation. One approach is to first isolate the linear sections of the ECG [30], and then remove the noise using a symmetric moving average linear-phase filter. The subtraction of the filtered and non-filtered segments results in an interference value. A similar procedure uses an added least squares and nonlinear regression routines to estimate sinusoidal parameters [31]. Other subtraction procedures utilize spline techniques to estimate the ECG signal [32].

Kelly and Calvert [33,34] introduced a simple and useful algorithm for removing sinusoidal interference from short data segments. Internally generated sine and cosine functions are essentially correlated with the recorded signal. When the frequency of the interference is known, the computed correlations, similar to Fourier series coefficients, are used to estimate the amplitudes of cosine and sine functions that are subtracted from the recorded signal. When the frequency is known only approximately, an algorithm can search in the neighborhood of the estimated frequency to find the minimum error, indicating the exact frequency of interference.

The Kelly-Calvert (K-C) method works well when the desired signal has little or no significant energy near the frequency of the interference. The method measures the combined amplitude of all components at a given frequency, and thus cannot distinguish between desired signal components and interference. When the interference frequency is not an integer number of cycles per data segment duration, the signal components at adjacent frequencies will be correlated with the reference sinusoids. Thus, they also contribute to the error in estimating the amplitude and phase of the interference.

Similar techniques have been applied in the context of a real digital system [35], including the use of oscillators to reduce memory requirements [36], and then the sinusoidal components and parameters are adjusted by the Wiener-Hopt equation of the system. This estimate is then followed by estimating the cross-correlations of the resulting Wiener-Hopt equation. Once the coefficients are found, the interference is subtracted [2].

This paper presents a new algorithm for calculating the 
amplitude and phase of the sinusoidal interference. Unlike the $\mathrm{K}-\mathrm{C}$ method, the new algorithm separates the signal from the sinusoidal interference. The only additional requirement is that the signal spectrum (without interference) be continuous at the frequency of the interference. The following describes the theoretical basis of the new algorithm and results of applying the new method to an ECG with various forms of interference. The $\mathrm{K}-\mathrm{C}$ method in included for comparison.

\section{Methods}

Consider an information-carrying noiseless signal $x(t)$ which has a Fourier Transform X( $\omega)$. The signal $x(t)$ could be complex, but for this discussion, $\mathrm{x}(\mathrm{t})$ is purely real. The recorded signal is represented as

$$
y(t)=x(t)+n(t)
$$

where $n(t)$ is an undesired or noise component. The additive noise could be a combination of random and deterministic components, but the focus is on a deterministic sinusoidal function whose amplitude and phase are unknown but whose frequency is known. Specifically, let

$$
n(t)=c \cos \left(\omega_{0} t\right)+s \sin \left(\omega_{0} t\right)
$$

so that the recorded signal is

$$
y(t)=x(t)+c \cos \left(\omega_{o} t\right)+s \sin \left(\omega_{o} t\right)
$$

The goal is to cancel the last two components of $y(t)$ such that $\mathrm{y}(\mathrm{t})$ only consists of $\mathrm{x}(\mathrm{t})$. To this end, consider $\mathrm{C}$ and $\mathrm{S}$, as initial amplitude estimates of $\mathrm{s}$ and $\mathrm{c}$ respectively. We can then calculate the desired function $\mathrm{z}(\mathrm{t})$ as

$$
z(t)=y(t)-C \cos \left(\omega_{o} t\right)-S \sin \left(\omega_{o} t\right)
$$

where $z(t)$ constitutes an estimation of $x(t)$. This can be extended to short discrete signals by simply replacing the continuous variable $\mathrm{t}$ by $\mathrm{nT}$, where $\mathrm{T}$ is the time interval between samples.

The function $X(\omega)$ and its derivative are assumed to be continuous at $\omega=\omega_{0}$, or that the real and imaginary parts of $X(\omega)$ and their derivatives both be continuous at $\omega=\omega_{0}$. This assumption is valid for most biomedical signals. In other words, $Y(\omega)$ is a smooth spectrum near $\omega=\omega_{0}$ except for an additive delta function at $\omega=\omega_{0}$. This is a quite realistic requirement for most time limited recorded signals.

If $\mathrm{Y}(\omega)$ is the Fourier Transform of $y(t)$, the derivative property of the Fourier Transform can be defined by the time-frequency equivalence

$$
\frac{d Y(\omega)}{d \omega} \leftrightarrow-j \operatorname{ty}(t)
$$

Applying Parseval's Theorem to the derivative of $\mathrm{Y}(\omega)$ in Equation 5,

$$
\int\left|\frac{d Y(\omega)}{d \omega}\right|^{2} d \omega=K \int\left|-j t y(t)^{2}\right| d t
$$

where the scaling factor $\mathrm{K}$ depends on the definition of the forward and inverse transform, and $\mathrm{K}$ is usually unity for the discrete transform. Both integrals can be referred to as mean squared values after normalization.

Given that $X(\omega)$ is continuous at $\omega_{0}$, non-zero positive or negative values of $\mathrm{c}$ or $\mathrm{s}$ will increase the left side of Equation 6. As a simple discrete-time example, consider a function $\mathrm{x}(\mathrm{nT})$ whose spectrum is linear, so that its derivative at $\omega_{0}$ is a constant $K$. Any non-zero $c$ or $s$ will introduce discontinuity $\mathrm{K} \pm \delta$ on either side of $\omega_{\mathrm{o}}$. In magnitude squared values, the sum of these two discontinuities is $2 \mathrm{~K}^{2}+$ $2 \delta^{2}$ compared with a value of $2 \mathrm{~K}^{2}$ when $\mathrm{s}$ and $\mathrm{c}$ are identically zero. Therefore, any positive or negative value of $\mathrm{c}$ or $\mathrm{s}$ contributes to an increase in the magnitude-squared value, giving rise to an integral that is minimum when $\mathrm{c}$ and $\mathrm{s}$ are both zero. As such, we have a mechanism for distinguishing the desired signal $\mathrm{x}(\mathrm{t})$ from the sinusoidal interference.

In the frequency domain, the spike at $\omega_{0}$ is graphically intuitive and the integral of the magnitude-squared value of the derivative on the left side of Equation 6 is also easily visualized. However, the right side of Equation 6 is computationally simpler and more efficient. Removing the sinusoidal interference corresponds to minimizing the left side of Equation 6, and by Parseval's Theorem, also minimizing the right side of Equation 6. Given the computational advantages, this method employs the time domain minimization.

Therefore, we can calculate

$$
J=\int|t y(t)|^{2} d t
$$

and find the values of $\mathrm{C}$ and $\mathrm{S}$ which minimize $\mathrm{J}$. From the previous argument, minima will occur when $\mathrm{C}=\mathrm{c}$ and $\mathrm{S}=\mathrm{S}$, provided that $X(\omega)$ ) is continuous at $\omega_{0}$.

For short segments of discrete signals, the integral in Equation 7 becomes a summation over the time of the entire segment. Therefore, the algorithm is relatively straightforward: for each data segment, $\mathrm{C}$ and $\mathrm{S}$ are varied to minimize $J$. In the case where $\omega_{0}$ is not known, it can be estimated by finding the frequency where $\mathrm{J}$ is minimum. A more straightforward method would be to make estimates for all three parameters, $C, S$, and $\omega_{\mathrm{O}}$ and minimize all three parameters as described above.

As a simple, continuous time example, consider the case when $x(t)=k \delta(t)$ which leads to $X(\omega)=k$. Therefore

$$
y(t)=k \delta(t)+c \cos (2 \pi f t)+s \sin (2 \pi f t)
$$

To illustrate the method, we choose to minimize $\mathrm{J}$, in Equation 7. This gives rise to

$$
J=\int|t k \delta(t)+t c \cos (2 \pi f t)+t \sin (2 \pi f t)|^{2} d t
$$


Employing the sifting property, and minimizing the result in Equation 9, shows that the minimum value of $\mathrm{J}$ occurs when the coefficients $\mathrm{c}=\mathrm{s}=0$.

\section{Results \& Discussion}

To demonstrate effectiveness of the new methods, a sample ECG signal from MIT-BIH physiological signals database [6] was loaded into the Matlab programming environment. A short segment of the signal was then corrupted with sinusoidal interference with amplitudes $\mathrm{s}=\mathrm{c}$ $=0.1$, a phase shift of 45 degrees, and varying frequencies. Figure 1 shows the original, noiseless cardiac cycle and the cardiac signal after being corrupted by $60 \mathrm{~Hz}$ PLI. It should be noted that the algorithm performance is invariant of interference amplitude and phase. The amplitude and phase invariance of our algorithm is critical in most bioelectric signal acquisition applications where the exact amplitude and phase characteristics of the noise are not known.

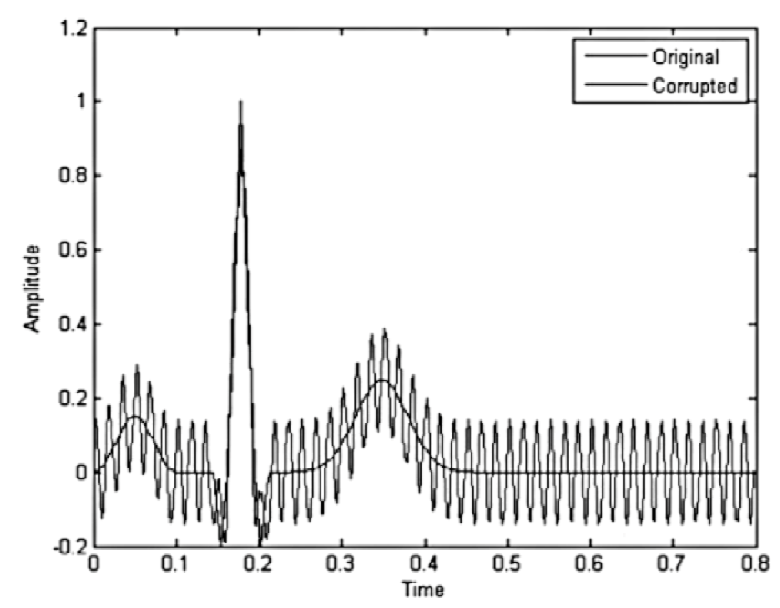

Figure 1. Original ECG signal and corrupted ECG signal

For each frequency, the Kelly-Calvert subtraction method and the new subtraction method are performed. Figure 2 shows the rms error vs. frequency for both methods. As it can be seen, the reconstruction of the corrupted ECG signal with the new method has a lower rms error over the entire frequency range, especially at the critical frequencies of 50 and $60 \mathrm{~Hz}$ for European and American power distribution systems respectively.

A correlation coefficient was then calculated to compare both reconstructed signals to the original signal, giving a measure of accuracy of reconstruction vs. frequency of interference. This is shown in Figure 3. Notice that the new method results in higher correlation coefficients over the entire frequency range, and shows significant improvement over the K-C method at lower frequencies.

Next, consider the case where the interference frequency is also unknown. This scenario will occur when power line frequencies drift or in other sinusoidal noise that is not from line interference. As discussed earlier, an initial guess is made, and a frequency range of the interference is determined. Applying the new method over the frequency range results in a global minimum in rms error occurring at the frequency of interference. Figure 4 demonstrates the algorithm finding the correct frequency of interference, in this case, $61.25 \mathrm{~Hz}$.

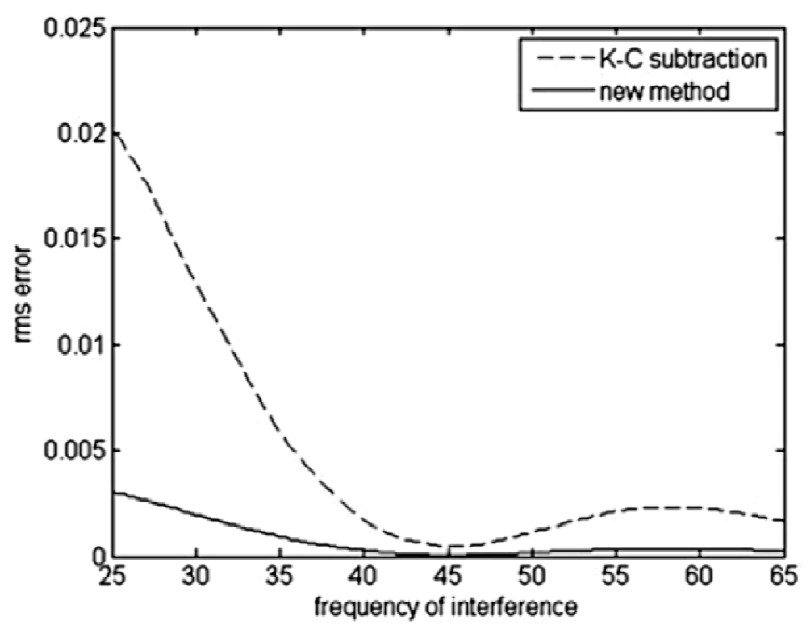

Figure 2. Rms error vs. frequency for both the Kelly-Calvert method and the new method.

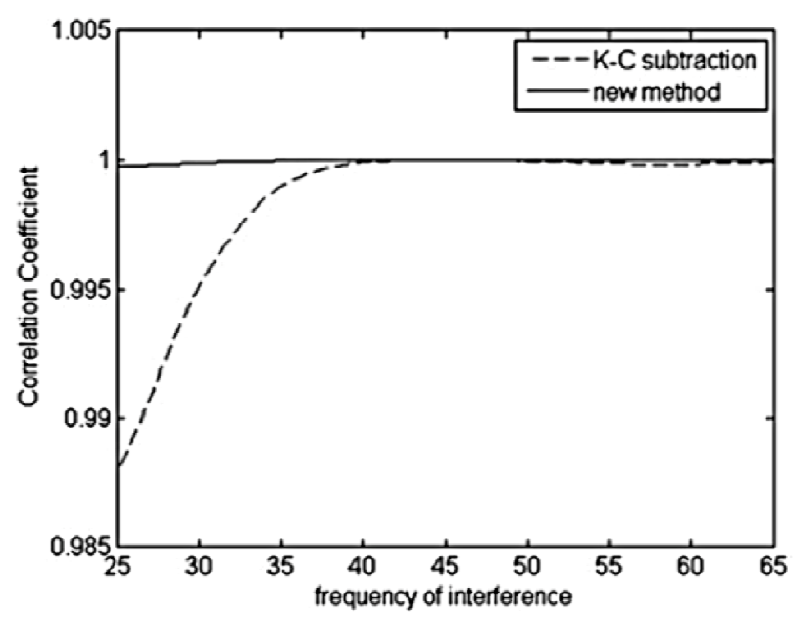

Figure 3. Correlation Coefficients for the Kelly-Calvert and new methods.

In many cases, the ECG will be corrupted by multiple sinusoids of varying amplitude, phase, and frequency. If the frequencies of interference are not known, they can be found from the local minima in rms error. Consider an ECG signal with interference from two sinusoids as shown in Figure 5. Two is chosen for convenience, but the method can be extended to multiple interference sinusoids as long as the interference frequencies do not lie extremely close to each other. The plot shown in Figure 6 demonstrates that the two sinusoidal interference frequencies occur at local minimums of the rms error function. With the interference frequencies now known, the original ECG signal can be completely reconstructed. 


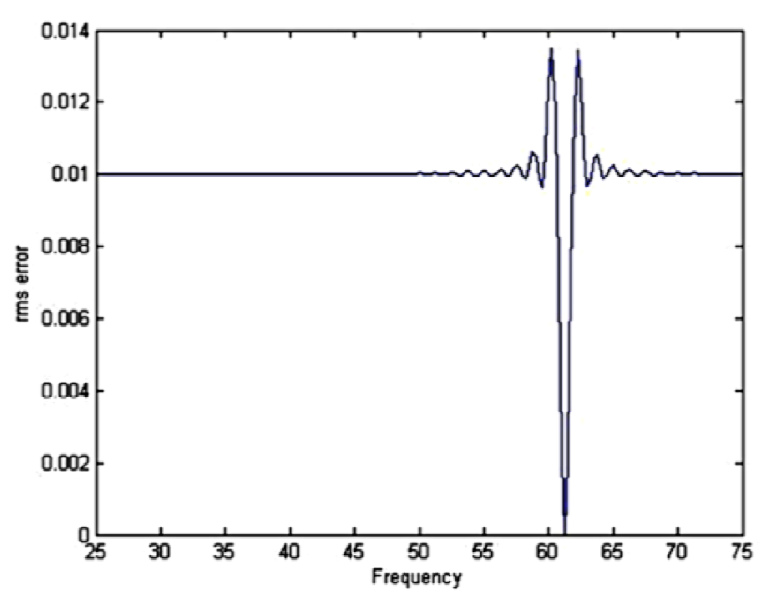

Figure 4. The minimum rms error occurs at $61.25 \mathrm{~Hz}$, which is the frequency of sinusoidal interference.

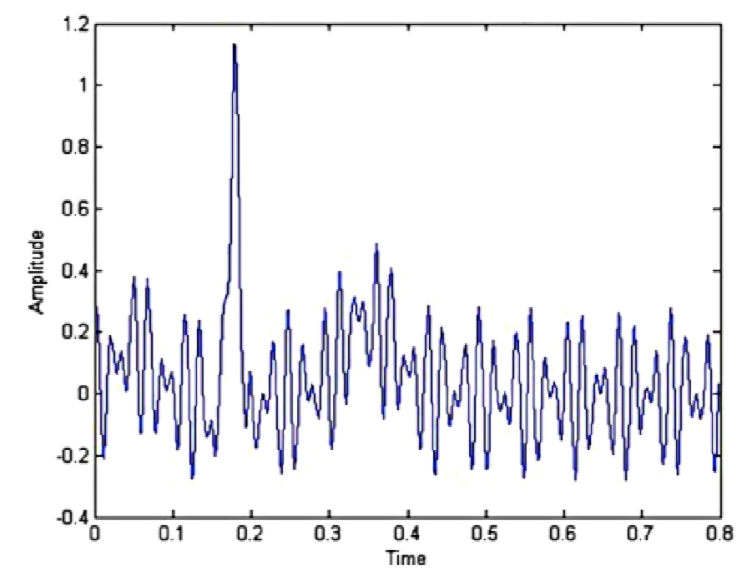

Figure 5. ECG corrupted with two sinusoids at 45 and $61.25 \mathrm{~Hz}$.

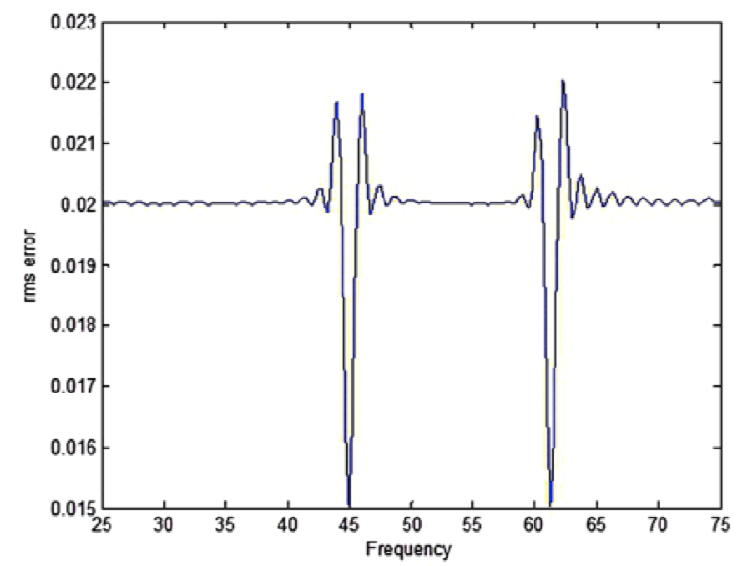

Figure 6. The frequencies of interference correspond to local minimums of the rms error of the new method.

\section{Conclusions}

Subtraction methods, due to their high efficiency and zero settling time, are ideal in digital applications where short digital segments are processed. The new method, based on Parseval's theorem and the derivative property of the Fourier transform, uses a time domain technique that effectively separates signal from interference. While this paper demonstrates the algorithms effectiveness on the ECG, the new method is applicable to any short data segment with a signal spectrum that is continuous at the frequency of the interference. The accuracy of this new method is not dependent on the relative phase or amplitude of the interference. The method does not require an integer number of cycles of interference in the data window because the time-domain computation avoids the fft artifact known as leakage. If the frequency of interference is not known, a local minimization procedure applied to the data window will produce a minimum at the frequency of interference, allowing the algorithm to determine individual interference frequencies.

When applied to the ECG, the new method provides excellent noise cancelation, offering near complete signal reconstruction. The algorithm is slightly perturbed by random noise as noise introduces slight discontinuities in the spectrum. However, the integral or sum computation has an inherent averaging effect on the noise, thereby limiting the error. A quantitative assessment of the noise effects and the errors due to uncertainty in $\omega_{\mathrm{O}}$ are subjects of further study. Additionally, this method can form the basis of an adaptive filter for use in long data segments, where the time-domain equivalent of the mean squared derivative in the frequency domain could be integrated into the traditional least squares structure.

\section{References}

[1] Chimene MF, Pallas-Areny R, A comprehensive model for power line interference in biopotential measurements. IEEE Transactions on Instrumentation and Measurement 2000, 49:535-540.

[2] Mewett DT, Nazeran H, Reynolds KJ, Removing power line noise from recorded EMG. Proc. $23^{\text {rd }}$ Annual Conference-IEEE-EMBS 2001, 3:2190-2193.

[3] Ortolan RL, Mori RN, Pereira, Jr. RR, Cabral CMN, Pereira JC, Cliquet, Jr. A, Evaluation of adaptive/nonadaptive filtering and wavelet transform techniques for noise reduction in EMG mobile acquisition equipment. IEEE Transactions on Neural Systems and Rehabilitation Engineering 2003, 11:60-69.

[4] Clifford G, Azuaje F, McSharrg P, The Physiological Basis of the Electrocardiogram. Advanced Methods and Tools for ECG Data Analysis, Aretech House: 2006.

[5] Sheikh RR, Taj IA, Cardiac disorder diagnosis based on ECG segments analysis and classification. Third International Conference on Electrical Engineering 2009, 00:1-6.

[6] Goldberger AL, Amaral LAN, Glass L, Hausdorff JM, Ivanov PC, Mark RG, Mietus JE, Moody GB, Peng CK, Stanley HE, PhysioBank, PhysioToolkit, and PhysioNet: Components of a New Research Resource for Complex Physiologic Signals. Circulation 2000, 101(23):e215-e220.

[7] Thomas CW, Huebner WP, Leigh RJ, A Low-Pass Notch Filter for Bioelectric Signals. IEEE Transactions On Biomedical Engineering 1988, 35:496-498. 
[8] Patel AJ, Patel V, Nyquist Filter Design to Remove Power Line Interference from Bio-Electric Signals. International Journal of Engineering Trends and Technology 2012, 3:81-83.

[9] Thomas C, Rzeszotarski M, Isenstein B, Signal averaging by parallel digital filters. IEEE Transactions on Acoustics, Speech and Signal Processing 1982, 30:338-346.

[10] Rompelman O, Ros HH: Coherent averaging technique, A tutorial review Part 2: Trigger jitter, overlapping responses and non-periodic stimulation. Journal of Biomedical Engineering 1986, 8:30-35.

[11] Rompelman O, Ros HH, Coherent averaging technique, A tutorial review Part 1, Noise reduction and the equivalent filter. Journal of Biomedical Engineering 1986, 8,24-29.

[12] Iravanian S, Tung L, A novel algorithm for cardiac biosignal filtering based on filtered residue method. IEEE Transactions on Biomedical Engineering 2002, 49,1310-1317.

[13] Burns M, Improving DSP-based measurements with spectral interpolation. Proc. International Test Conference 1995, 00:355-363.

[14] Vullings R, de Vries B, Bergmans JWM, An Adaptive Kalman Filter for ECG Signal Enhancement. IEEE Transactions on Biomedical Engineering 2011, 58,1094-1103.

[15] Li G, Zhang S, Xiao-jie Hao L, Zhou X, Robust power line interference suppression for ECG signal based on $\mathrm{H}$ filter. International Symposium on Bioelectronics and Bioinformatics(ISBB) 2011, 00:143-146.

[16] McNamara DM, Majo JP, Ziarani AK, Elimination of Power Line Interference on Telephone Cables Under Frequency-Varying Conditions. IEEE Transactions on Instrumentation and Measurement 2008, 57:321-331.

[17] Ziarani AK, Konrad A, A nonlinear adaptive method of elimination of power line interference in ECG signals. IEEE Transactions on Biomedical Engineering 2002, 49:540-547.

[18] Martens SMM, Mischi M, Oei SG, Bergmans JWM, An Improved Adaptive Power Line Interference Canceller for Electrocardiography. IEEE Transactions on Biomedical Engineering 2006, 53:2220-2231.

[19] Jiang Y, Ma W, and Zhao Z, The analysis of an adaptive sinusoidal interference cancellation system. International Conference on Electrical Machines and Systems 2008, 00:640-645

[20] Mallat S, Sparse Representations. A Wavelet Tour of Signal Processing, $1^{\text {st }}$. Burlington, MA: Elsevier; 2009.

[21] Mahmoodabadi AASZ, Abolhasani MD, ECG Feature Extraction Using Daubechies Wavelets. Proc. of the Fifth IASTED International Conference Visualization, Imaging, and Image Processing 2005, 00:343-348.

[22] Vetterli M, Kovačević J, Goyal VK, Signal Processing: Fourier and Wavelet Representations. Online. Available: http://www.fourierandwavelets.org.
[23] $\mathrm{Xu} \mathrm{L}$, Cancellation of harmonic interference by baseline shifting of wavelet packet decomposition coefficients. IEEE Transactions on Signal Processing 2005, 53:222-230.

[24] Zhang W, Wang X, Ge L, Zhang Z, Noise Reduction in ECG Signal Based on Adaptive Wavelet Transform. Proc. IEEE/EMBS Annual Conference 2005, 00:2699-2702.

[25] Tikkanen P, Nonlinear wavelet and wavelet packet denoising of electrocardiogram. Biological Cybernetics 1999, 80:259-267.

[26] Ando S, Nara T, An Exact Direct Method of Sinusoidal Parameter Estimation Derived From Finite Fourier Integral of Differential Equation. IEEE Transactions on Signal Processing, 57:3317-3329.

[27] O. Paiss, Elimination of exponential interference form finite-length discrete signals. IEEE Transactions on Acoustics, Speech and Signal Processing 1990, 38:2189-2191.

[28] B. Kwan, K. Ong, and R. Paramesran, Noise Removal of ECG Signals using Legendre Moments. Proc. IEEE/EMBS Annual Conference 2005, 00:5627-5630.

[29] Levkov C, Mihov G, Ivanov R, Daskalov I, Christov I, Dotsinsky I, Removal of power-line interference from the ECG: a review of the subtraction procedure. BioMedical Engineering OnLine 2005, 4:50.

[30] Mihov G, Subtraction procedure for removing powerline interference from ECG. $4^{\text {th }}$ International Conference on Biomedical Engineering and Informatics (BMEI) 2011, 00:858-861.

[31] $\mathrm{Hu} \mathrm{X,} \mathrm{Xiao} \mathrm{Z,} \mathrm{Liu} \mathrm{C,} \mathrm{Reduction} \mathrm{arithmetic} \mathrm{for} \mathrm{power} \mathrm{line}$ interference from ECG based on estimating sinusoidal parameters. in $3^{\text {rd }}$ International Conference on Biomedical Engineering and Informatics (BMEI) 2010, 00:2089-2092.

[32] Caroubalos C, Perche C, Metaxaki-Kossionides C, Sangriotis E, Maroulis D, Method for an automatic analysis of the ECG. J. Biomed Eng. 1988, 10:343-347.

[33] Kelly KK, Calvert TW, The Removal of Coherent Noise from Short Digitized Records. IEEE Transactions on Biomedical Engineering 1970, BME-17:78-78.

[34] Walter DO, Kelly KK, Calvert TW, Comments on The Removal of Coherent Noise from Short Digitized Records. IEEE Transactions on Biomedical Engineering 1970 BME-17, BME-17:357-359.

[35] Köymen H, Ider YZ, Hizarci G, Kucukdeveci F, Tuzun F, Yaylali I, A novel quadrature interference subtraction technique in ECG noise reduction. Proc. Computers in Cardiology 1988, 00:409-412.

[36] Nosan A, Punchalard R, Wardkein P, Koseeyaporn J, Novel algorithm for sinusoidal interference cancellation. International Symposium on Intelligent Signal Processing and Communications Systems (ISPACS) 2011, 00:1-4. 